\title{
The Microstructure of Dislocated Martensitic Steel: Microscopy and Microanalysis
}

\author{
J.W. Morris Jr. ${ }^{1}$. \\ 1. Dept. Materials Science and Engineering, Univ. of California, Berkeley, CA, USA
}

My purpose in this paper is to provide a brief historical review of progress in elucidating the microstructure of dislocated (lath) martensitic steel, a subject that greatly interested Gareth Thomas, and was the focus of a large part of his research and a significant fraction of his technical publications over the course of his career. Transmission electron microscopy played a significant role, along with other characterization techniques, in research on this problem. It remains only partly solved, though significant progress has been made.

Until the late 1960's microstructural studies of dislocated martensite had relied on optical metallography along with the low-resolution x-ray techniques that were available at the time. The main product if that research was to reveal the complexity of the problem. Optical metallography showed that the lath martensite $\left(\alpha^{\prime}\right)$ that developed within a transformed prior austenite $(\gamma)$ grain was a very fine-grained mixture of extended laths or plates with various spatial orientations. There were identifiable assemblages of parallel plates that were called "packets," these were often subdivided into semi-distinct bands that were termed "blocks" and these were, in turn, assemblages of individual laths of microscopic size. The most important result of the x-ray work was to identify the "Kurdjumov-Sachs" crystallographic relations between the $\alpha$ ' and the parent $\gamma$ phases. These showed that the phases had one close-packed plane and one close-packed direction in common. However, there are 24 different ways to satisfy these constraints, hence 24 different crystallographic variants that satisfy the KS relations. It was not at all clear how many if these appeared in the typical transformed austenite grain or how they were selected. The x-ray data had also showed that as-quenched lath martensite often retained a measurable fraction of the high-temperature $\gamma$ phase, particularly when the carbon content was appreciable.

The application of high-resolution electron microscopy to the microstructure of lath martensitic steel accelerated in the late 1960's, through the work of Gareth Thomas and many others, and led to a host of important insights. Among the most elementary and important was the recognition that the laths in the relatively low-carbon steels that dominated structural applications were heavily dislocated, rather than twinned as their high-carbon counterparts were. The dislocated laths were, indeed, hyperfine, with narrow dimensions often no more than a fraction of a micron.

Focusing specifically on the contributions of TEM to understanding the structure of lath martensitic steel, there were several important results. First, though bright-field TEM revealed an ultra-fine grained structure of dislocated laths, local-area diffraction showed that these observations were deceptive. The well-defined boundaries between adjacent laths were, ordinarily, such low-angle boundaries that microvolumes of significant size were so aligned in their crystallography as to behave mechanically as single crystals. These self-aligned subvolumes appeared to correspond to the "blocks" that had been identified through optical metallography. The crystallographic evidence suggested that the size of these aligned blocks determined the "effective grain size" of the steel. The blocks cleaved as a unit below the ductile-brittle transition temperature, and deformed under load as if they were single-crystal grains.

While the lath boundaries within the blocks were invariably low-angle boundaries, high-resolution 
microscopy showed that they were, nonetheless, active in setting the microstructure and controlling many of the processes that were used to modify it in beneficial ways. For example, high-resolution TEM revealed the presence of films of retained austenite along the lath boundaries of as-quenched higher-carbon martensites (for example, in 4340 and similar high-strength structural steels). These sheaths of interlath austenite proved beneficial to properties such as tensile elongation via their straininduced transformation (TRIP) under load. On the other hand, their decomposition during tempering at relatively low temperature was shown to create sheaths of carbide precipitate that led to a "tempered martensite embrittlement" that is sometimes catastrophic.

High-resolution TEM studies of laths and their boundaries also showed how they might be used to tailor the microstructure of the martensite blocks to control properties. In particular, re-heating the steel triggered the precipitation of austenite phase along the lath boundaries, which was used to develop three important processes: (1) A treatment at a low temperature within the two-phase region (intercritical tempering) precipitates thermally stable austenite along the lath boundaries. This austenite breaks up the alignment of laths within the block and dramatically improves low-temperature toughness. It is widely used to process steels for low-temperature use. (2) A treatment at a higher temperature within the twophase region (intercritical annealing) produces interlath austenite that re-transforms on cooling. The result is "dual-phase" steel, with blocks in which laths of fresh martensite alternate with laths of welltempered martensite. These steels have tailored work-hardening characteristics that produce very good tensile elongation. They are widely used in the automotive industry. (3) A treatment that uses an intercritical anneal followed by reversion to the austenite phase and subsequent quenching (the "QLQ" treatment) produces a dislocated martensite structure in which adjacent laths are different KS variants of the martensite, leading to exceptional combinations of strength and toughness. Many useful modifications of these basic treatments have been developed or proposed on the basis of detailed analysis of the microstructural modifications they accomplish.

Many other advances in scientific understand and useful technology were also achieved through highresolution TEM, particularly concerning the structure, distribution and control of hardening precipitates and the microstructure of alternative structures, such as the various forms of ferritic and bainitic constituents in steel.

What optical metallography and high-resolution TEM could not do, alone or together, however, was to characterize and understand the overall microstructure of dislocated martensitic steel. TEM operates at too high a magnification to fully image prior austenite grains in a useful manner. Optical metallography images grains, but provides no crystallographic information. Hence useful clarification of the overall microstructure of dislocated lath martensitic steel did not happen until the development of new characterization techniques, specifically including electron backscatter diffraction (EBSD) in the scanning electron microscope. EBSD allows combined morphological and crystallographic characterization over a range of magnification from that typical of optical metallography to that used in low-resolution TEM. The exploitation of this technique has finally allowed a reasonably complete understanding of the microstructural pattern of dislocated martensitic steel and the mechanisms that cause this pattern to form. 